\title{
MORTALITY OF VARIOUS LEPIDOPTERAN LARVAE INFECTED BY NEW ZEALAND ZOOPHTHORA RADICANS ISOLATES FROM DIFFERENT HOSTS
}

\author{
M. WALTER ${ }^{1}$, F.J.L. STAVELY ${ }^{1}$, R.B. CHAPMAN ${ }^{2}$, J.K PELL ${ }^{3}$, \\ T.R. GLARE ${ }^{4}$, P.A. ALSPACH ${ }^{5}$ and S.M. ZYDENBOS ${ }^{6}$ \\ ${ }^{1}$ HortResearch, P.O. Box 51, Lincoln, New Zealand \\ ${ }^{2}$ Soil, Plant and Ecological Sciences Division, PO Box 84, \\ Lincoln University, Canterbury \\ ${ }^{3}$ Rothamsted Research, Harpenden, Hertfordshire, AL5 2JQ, United Kingdom \\ ${ }^{4}$ Biocontrol and Biosecurity Group, AgResearch, PO Box 60, Lincoln, New Zealand \\ ${ }^{5}$ HortResearch, P.O. Box 220, Motueka, New Zealand \\ ${ }^{6}$ Old West Coast Road, Halkett, RD 1, Christchurch, New Zealand \\ Corresponding author: mwalter@hortresearch.co.nz
}

\begin{abstract}
Zoophthora radicans, an entomophthoralean fungus, is a potential biocontrol agent for a wide range of insect pests. The mortality of six insect species inoculated with twelve $Z$. radicans isolates from different hosts found in New Zealand was evaluated using a bioassay. Zoophthora radicans isolates originating from the host being tested were generally, but not always, more effective than isolates originating from other species. For example, lightbrown apple moth (LBAM) was highly susceptible to isolates Z2 and Z6 from leafrollers (96\% and 89\% mortality respectively), but was not susceptible to any isolates from diamondback moth (DBM) (mortality $<5 \%, \mathrm{P}<0.001$ ). DBM was highly susceptible to all isolates from DBM ( $>96 \%$ mortality) but only moderately susceptible to leafroller isolates $(<66 \%, \mathrm{P}<0.001)$. Although resting spore production was low overall, some isolates produced more resting spores in certain hosts, such as Z2 and Z6 in LBAM $(\mathrm{P}<0.001)$ and Z8 and Z5 in DBM $(\mathrm{P}<0.05)$.
\end{abstract}

Keywords: lightbrown apple moth, diamondback moth, greenheaded leafroller, brownheaded leafroller, biocontrol.

\section{INTRODUCTION}

Zoophthora radicans (Brefeld) Batko is an entomophthoralean fungus that has been recorded from a wide range of insect orders. Many economically important insects that attack vegetable and horticultural crops, such as diamondback moth (DBM, Plutella xylostella (L.)), lightbrown apple moth (LBAM, Epiphyas postvittana (Walker)), greenheaded leafroller (GHLR, Planotortrix spp.) and brownheaded leafroller (BHLR, Ctenopseustis obliquana (Walker)), may be infected by Z. radicans. Pell et al. (2001) reviewed the host specificity of $Z$. radicans and concluded that there are various pathotypes of the fungus, each with a limited host range.

Zoopthora radicans is capable of producing several types of spores (conidia). The life cycle of the pathogen involves two stages. Firstly there is an epizootic phase, where short-lived, forcibly discharged primary conidia either directly infect new hosts or produce secondary conidia that can infect hosts. The second is a survival stage, where thickwalled resting spores (zygospores or azygospores) are produced within cadavers. The resting spores are capable of surviving for several years in protected environments. Like all entomophthoralean fungi, $Z$. radicans is sensitive to environmental conditions for infection of host insects. High humidity, close to $100 \% \mathrm{RH}$, is required for conidial germination and sporulation (Pell et al. 2001). Temperature can also be a limiting factor in development of disease in hosts. 
To develop Z. radicans as an effective biocontrol agent, methods for infecting host insects must be developed. Milner et al. (1982) achieved infection of spotted alfalfa aphid (Therioaphis trifolii (Monell) f. maculata) in the field by placing sporulating cultures of $Z$. radicans over lucerne plants infected with aphids or by releasing laboratoryinfected aphids. Subsequent survival of the fungus through periods without hosts or during winter was through resting spores.

The present laboratory study evaluated insect mortality and resting spore production of twelve New Zealand Z. radicans isolates in six insect hosts. Effects of various temperature and photoperiod conditions, as well as host and inoculum age were determined.

\section{Zoophthora radicans isolates}

\section{MATERIALS AND METHODS}

Twelve $Z$. radicans isolates, designated $\mathrm{Z1-Z12}$, were used in this study. Z1 was recovered from Australian flat moth (AFM) (Eutorna phaulacosma Meyrick); Z3, Z4, Z5, Z7, Z9, Z10, Z11 and Z12 were isolated from DBM; Z2 and Z6 were isolated from unidentified leafroller caterpillars; and Z8 was isolated from tomato fruitworm (TFW) (Helicoverpa armigera conferta Walker). The isolates Z4, Z10 and Z12 corresponded to isolate codes NW328, NW334 and NW337, respectively, used in earlier studies (Yeo et al. 2001). Isolates were maintained as described by Yeo et al. (2001). Zoophthora radicans inoculum was produced by growing the fungi for $1-2$ weeks $\left(20^{\circ} \mathrm{C}\right)$ in the dark on Sabouraud Dextrose Yeast Agar (SDYA) containing 65 g Sabouraud dextrose agar (Difco) with $6.5 \mathrm{~g}$ yeast extract (Merck) per litre distilled water.

\section{Insects}

Six species of lepidopteran larvae were used in these experiments. They were lightbrown apple moth (LBAM), two species of greenheaded leafrollers (GHLR, Planotortrix octo Dugdale and Planotortrix excessana (Walker)), brownheaded leafroller (BHLR), diamondback moth (DBM) and TFW. DBM larvae were reared on organicallygrown cabbage leaves and all other larvae were reared on general purpose diet (GPD) (Singh 1983) at $20^{\circ} \mathrm{C}, 12: 12 \mathrm{~h}$ light:dark photoperiod. Larvae were fed on standard GPD up to 2 days prior to exposure to $Z$. radicans inoculum and then on fungicide-free GPD that had been stored at $-20^{\circ} \mathrm{C}$ to avoid spoilage. Larvae used in experiments were second or third instars, except in experiment 7 (E7) where pre-pupal larvae were included for comparison.

\section{Bioassay}

The basic methods previously described by Yeo et al. (2001) were modified for these experiments. From colonies grown on SDYA, 24 agar plugs (4 mm diameter) were cut and evenly placed in a grid pattern, myrelial side up, in $90 \mathrm{~mm}$ diameter Petri dishes containing $1 \%$ water agar (WA; Merck). The WA plates were then incubated for 2 days (referred to as 2-day-old inoculum, 2DI) at $20^{\circ} \mathrm{C}$ in the dark to induce discharge of primary conidia. In one experiment (E6) the plates were left one extra day before showering (3DI). Following 2 or 3 day incubation of the agar plugs, conidia were showered onto new WA plates for $1 \mathrm{~h}$ under high humidity. A glass coverslip placed on the plate during inoculation was used to estimate density of conidium populations as described by Yeo et al. (2001). These plates were then incubated in the dark at $20^{\circ} \mathrm{C}$ at $100 \% \mathrm{RH}$ for approximately $16 \mathrm{~h}$ to allow production of capilliconida (sessile secondary conidia formed from the primary conidia). In the centre of each capilliconidiumcontaining Petri dish, 10-15 larvae were placed and allowed to migrate to the side of the Petri dish to collect capilliconidia. Larvae were then removed individually (aseptically) to clean Petri dishes containing food and left at $20^{\circ} \mathrm{C}$ and $100 \% \mathrm{RH}$ in the dark for a further $24 \mathrm{~h}$ to encourage infection. All surviving larvae were then transferred to ventilated Petri dishes with food. Food consisted of cabbage leaf pieces for DBM larvae and fungicide-free GPD for all other larvae. Food was changed at 1-2 day intervals. For the next 5 days dead larvae were removed daily, incubated, and then examined for fungal sporulation and presence of resting spores. The proportion of each cadaver containing resting spores (area of infection) was estimated as falling into one of the following categories: $0,1-10 \%, 10-50 \%, 50-75 \%, 75-90 \%$ or $100 \%$. 


\section{Experimental design and statistical analysis}

Twelve separate experiments (E1-E12) examined the effects of different strains of $Z$. radicans on the mortality of various insect larvae (Table 1). In some experiments different conditions of temperature and light were used, while E7 used a different larval stage and E6 compared two ages of inoculum. All experiments were randomised complete block designs with five replicates; a replicate being a single Petri dish with 10-15 larvae. In E9 there were insufficient larvae so some treatments only had four replicates. Control treatments, consisting of 10-15 larvae on WA plates that had not been inoculated with fungus, were used for all experiments except for E3 and E6 which were carried out at the same time as E4, for which there were controls.

TABLE 1: Summary of the twelve experiments testing various strains of $Z$. radicans on different host species under various temperature, photoperiod, host age and inoculum conditions.

\begin{tabular}{lcccccc}
\hline Expt & Strains & Host species & $\begin{array}{c}\text { Photo } \\
\text { period }\end{array}$ & $\begin{array}{c}\text { Temperature } \\
\left({ }^{\circ} \mathrm{C}\right)\end{array}$ & $\begin{array}{c}\text { Inoculum } \\
\text { age }(\text { days })\end{array}$ & $\begin{array}{c}\text { Host } \\
\text { age }\end{array}$ \\
\hline E1 & Z1-Z8 & LBAM & $16: 8$ & 20 & 2 & 23IN \\
E2 & Z1-Z8 & LBAM & $16: 8$ & 20 & 2 & 23IN \\
E3 & Z1, Z2 \& Z6 & LBAM & $16: 8 \& 12: 12$ & 20 & 2 & 23IN \\
E4 & Z1, Z2 \& Z6 & LBAM & $16: 8$ & $5,15 \& 25$ & 2 & 23IN \\
E5 & Z1, Z2 \& Z6 & LBAM & $16: 8$ & $5,15 \& 25$ & 2 & 23IN \\
E6 & Z1 & LBAM & $16: 8$ & 20 & $2 \& 3$ & 23IN \\
E7 & Z1, Z2 \& Z6 & LBAM & $16: 8$ & 20 & 2 & PPL \\
E8 & Z1-Z8 & DBM & $16: 8$ & 20 & 2 & 23IN \\
E9 & Z1-Z3 \& Z8-Z12 & P. excessana & $16: 8$ & 20 & 2 & 23IN \\
E10 & Z1-Z8 & P. octo & $16: 8$ & 20 & 2 & 23IN \\
E11 & Z1-Z8 & BHLR & $16: 8$ & 20 & 2 & 23IN \\
E12 & Z1-Z8 & TFW & $16: 8$ & 20 & 2 & 23IN \\
\hline
\end{tabular}

${ }^{1}$ Second and third instar larvae were used, except in E7 where pre-pupal larvae (PPL) were used.

Data of mortality and conidium concentration were examined by analysis of variance, after excluding treatments with 0 or $100 \%$ mortality that would violate the homoscedasicity assumption. Mean conidium concentration was similarly analysed, although a logarithmic transformation was necessary for data from E8 and E12. Mortality from E1, E8 and E12 was analysed with mean conidium concentration as a covariate, fitted both before and after the strain. Residual plots were examined to check that normality and homoscedasicity assumptions were not grossly violated.

\section{RESULTS}

Mortality of the six larval species tested for susceptibility to the twelve $Z$. radicans isolates is shown in Table 2. Data for LBAM were from E2, which was typical of the six other experiments involving LBAM. LBAM was highly susceptible to isolates Z2 and Z6 from unidentified leafrollers and Z1 from AFM, but was not killed by Z. radicans from DBM or TFW ( $\mathrm{P}<0.001)$. In contrast, DBM was highly susceptible to all isolates of $Z$. radicans from DBM and the isolate from TFW (P<0.001). DBM mortality was moderate when inoculated with $Z$. radicans from AFM (Z1) and one leafroller (Z2), while Z6 only gave low mortality. Mortality was low to moderate for both GHLRs, $P$. octo and $P$. excessana, with all isolates of $Z$. radicans tested. Only isolates from DBM gave moderate mortality (Z5 for $P$. octo and Z9, Z10 and Z12 for P. excessana). BHLR was highly susceptible to $Z$. radicans from AFM, moderately to highly susceptible to the two isolates from leafrollers (Z2 and Z6) and two isolates from DBM (Z4 and Z5) and moderately susceptible to the isolate from TFW (Z8) and another DBM isolate 
(Z7). BHLR was not susceptible to Z3 from DBM. Surprisingly, TFW was not susceptible to $Z$. radicans isolated from its own species. However, in this experiment (E12) there was significant cannibalism with only low mortality from any of the $Z$. radicans isolates, so the results should be interpreted with caution.

Mortality of LBAM did not differ between the various temperature and photoperiod treatments in E3, E4 and E5, and pre-pupal LBAM mortality was similar to mortality for second and third instar larvae (E7). However, in E6 3-day-old inoculum was less effective than 2-day-old inoculum ( $40 \%$ versus $74 \%$, respectively, SED $=14.8 \%$ ). In covariate analysis, conidium concentration fitted before isolate had a significant effect on mortality in E1 and E8 $(\mathrm{P}<0.01)$ but the isolate effect was still significant $(\mathrm{P}<0.001)$. When conidium concentration was fitted as a covariate after fitting isolate, it had no effect $(\mathrm{P}>0.05)$ on mortality.

TABLE 2: Mortality (\%) of various larvae due to $Z$. radicans isolated from different host species. The experiment number is shown in parentheses.

\begin{tabular}{lrrrrrrr}
\hline & \multicolumn{7}{c}{ Larval species tested } \\
\cline { 2 - 8 } Source of Z. radicans & $\begin{array}{r}\text { LBAM } \\
(\mathrm{E} 2)\end{array}$ & $\begin{array}{r}\text { DBM } \\
(\mathrm{E} 8)\end{array}$ & $\begin{array}{r}\text { P. excessana } \\
(\mathrm{E} 9)\end{array}$ & $\begin{array}{r}\text { P. octo } \\
(\mathrm{E} 10)\end{array}$ & $\begin{array}{r}\text { BHLR } \\
(\mathrm{E} 11)\end{array}$ & $\begin{array}{r}\text { TFW } \\
(\mathrm{E} 12)\end{array}$ \\
\hline AFM & Z1 & 73 & 75 & 9 & $0^{1}$ & 95 & 37 \\
Leafroller & Z2 & 96 & 66 & 24 & 11 & 74 & 21 \\
Leafroller & Z6 & 89 & 27 & $\mathrm{nd}^{2}$ & 6 & 71 & 20 \\
TFW & Z8 & $0^{1}$ & 94 & 17 & 12 & 42 & 11 \\
DBM & Z3 & $0^{1}$ & $100^{1}$ & 24 & 4 & 2 & 5 \\
DBM & Z4 & 3 & 96 & nd & 23 & 83 & 13 \\
DBM & Z5 & 2 & 98 & nd & 46 & 80 & 21 \\
DBM & Z7 & 5 & $100^{1}$ & nd & 3 & 64 & 11 \\
DBM & Z9 & nd & nd & 68 & nd & nd & nd \\
DBM & Z10 & nd & nd & 51 & nd & nd & nd \\
DBM & Z11 & nd & nd & 38 & nd & nd & nd \\
DBM & Z12 & nd & 98 & 67 & nd & nd & nd \\
\hline Control & & $0^{1}$ & 15 & 11 & 2 & 6 & 11 \\
SED & 5.7 & 9.0 & 17.1 & 7.4 & 11.0 & 11.0 \\
P-value & $<0.001$ & $<0.001$ & 0.007 & $<0.001$ & $<0.001$ & 0.235 \\
\hline
\end{tabular}

${ }^{1}$ Excluded from the statistical analyses.

${ }^{2}$ nd $=$ not determined.

Resting spores were produced in relatively few of the dead larvae so only experiments with LBAM, DBM and BHLR were analysed for this characteristic. In addition, any isolates with mortality below $70 \%$ were omitted from the analyses as these would not give meaningful results. Isolate $\mathrm{Z} 2$, originating from leafroller, produced the greatest proportion of larvae with resting spores in LBAM and BHLR (Table 3). Isolate Z6, also from a leafroller, gave a high proportion of larvae with resting spores in LBAM but not in BHLR. Production of resting spores in DBM was greatest from Z5 and Z8. Isolates $\mathrm{Z1}, \mathrm{Z3}, \mathrm{Z4}$ and Z7 produced very few resting spores. Those isolates that had a high proportion of dead larvae with resting spores tended to have a higher average area of infection (Pearson's correlation coefficient, $r=0.82$ ). 
TABLE 3: Percentage of dead larvae containing resting spores for three species inoculated with eight isolates of $Z$. radicans.

\begin{tabular}{|c|c|c|c|c|}
\hline \multirow{2}{*}{\multicolumn{2}{|c|}{ Source of Z. radicans }} & \multicolumn{3}{|c|}{ Larval species tested } \\
\hline & & \multirow{2}{*}{$\begin{array}{r}\text { LBAM } \\
2\end{array}$} & \multirow{2}{*}{$\begin{array}{c}\text { DBM } \\
0\end{array}$} & \multirow{2}{*}{$\begin{array}{c}\text { BHLR } \\
0\end{array}$} \\
\hline AFM & $\mathrm{Z1}$ & & & \\
\hline Leafroller & $\mathrm{Z} 2$ & 53 & 9 & 30 \\
\hline Leafroller & Z6 & 49 & omitted $^{1}$ & 0 \\
\hline TFW & Z8 & omitted & 24 & omitted \\
\hline DBM & $\mathrm{Z} 3$ & omitted & 0 & omitted \\
\hline DBM & Z4 & omitted & 0 & 3 \\
\hline DBM & $\mathrm{Z} 5$ & omitted & 22 & 8 \\
\hline DBM & $\mathrm{Z7}$ & omitted & 2 & 2 \\
\hline SED & & 9.5 & 6.5 & 9.5 \\
\hline P-value & & $<0.001$ & 0.015 & 0.045 \\
\hline
\end{tabular}

${ }^{1}$ Omitted from analysis as mortality below $70 \%$.

\section{DISCUSSION}

Mortality from infection by $Z$. radicans differed with the isolate used and the host species tested. In general, isolates originally from the particular insect species being tested were more effective than isolates from other species. For example, isolate Z6 from an unidentified leafroller was highly effective on LBAM and BHLR but gave only low mortality in DBM, while Z3, isolated from DBM, was highly effective on DBM but not on any other species. However, there were exceptions to this as Z1, which was isolated from AFM, gave moderate to high mortality in LBAM, DBM and BHLR. Mortality of TFW was low with Z8, isolated from its own species, but, as previously mentioned, there was significant cannibalism and the bioassay may need to be redesigned for this species to prevent this occurring. Previously, it has been established that $Z$. radicans occurs as pathotypes restricted to only some of the known host range for the fungus. For example, in Australia, indigenous strains of $Z$. radicans found on Lepidoptera and Diptera did not infect the exotic spotted alfalfa aphid (SAA; Therioaphis trifolii (Monell) f. maculata). However, a strain isolated from SAA in Israel was highly effective against Australian SAA in laboratory bioassays and the field (Milner et al. 1982; Milner \& Mahon 1985).

The lack of effect of conidium concentration when fitted to mortality data as a covariate after fitting the isolate effect, suggests that conidium concentration was not the only factor affecting efficacy. In fact, some highly effective isolates had relatively low conidium concentrations, which may be a useful characteristic for field infections. E6 indicated that efficacy is also influenced by inoculum age, which is most likely related to the relatively rapid production and discharge of primary conidia on water agar, and the lack of persistence of conidia after discharge.

Resting spores are likely to be a more robust source of inoculum than conidia and may be essential for maintaining long-term control by Z. radicans. Resting spores are produced in response to certain environmental factors, which are not well understood, and may not be produced in all isolates (Glare et al. 1989). Lower temperatures (in the range of $\left.10-25^{\circ} \mathrm{C}\right)$, high humidity $(>95 \% \mathrm{RH})$ and high inoculum density were positively correlated with resting spore production in strains tested previously (Glare et al. 1989). In the experiments described in the present paper, environmental conditions may not have been conducive to resting spore production, which was generally low. However, isolate $\mathrm{Z} 2$ produced resting spores in 53\% of LBAM and 30\% of BHLR cadavers, so further studies with this isolate are warranted. Resting spore production in DBM was best with isolates Z8 (24\%) and Z5 (22\%). These values are larger than those recorded for the four isolates tested on DBM by Yeo et al (2001). It is not known why no resting 
spores were produced in DBM infected by isolate Z4 in these experiments, while $8 \%$ of cadavers infected with the same isolate (named NW328) produced resting spores in the work of Yeo et al (2001). While environmental factors are important for resting spore production, there is also a strong genetic element, which results in the strain of fungus having more influence on the level of resting spore production than environment (Glare et al. 1989).

In conclusion, for inducing high mortality and producing resting spores, isolate $\mathrm{Z} 2$ was most effective for LBAM and BHLR, while isolate Z5 was most effective for DBM. These isolates require further testing in the laboratory to determine the environmental and genetic factors that regulate resting spore production. Resting spore production will be required in any isolate released as a control agent, to enable the fungus to persist between seasons and during unfavourable conditions.

\section{ACKNOWLEDGEMENTS}

This research was funded by the New Zealand Foundation for Research, Science and Technology. We thank Ann Barrington and Graham Walker for supply of insect larvae and eggs.

\section{REFERENCES}

Glare, T.R.; Milner, R.J.; Chilvers, G.A. 1989: Factors affecting the production of resting spores by Zoophthora radicans in the spotted alfalfa aphid Therioaphis trifolii $\mathrm{f}$. maculata. Can. J. Bot. 67:848-855.

Milner, R.J.; Mahon, R. 1985: Strain variation in Zoophthora radicans a pathogen of a variety of insects in Australia. J. Aust. Entomol. Soc. 24: 195-198.

Milner, R.J.; Soper, R.S.; Lutton, G.G. 1982: Field release of an Israeli strain of the fungus Zoophthora radicans (Brefeld) Batko for biological control of Therioaphis trifolii (Monell) f. maculata. J. Aust. Entomol. Soc. 21: 113-118.

Pell, J.K.; Eilenberg, J.; Hajek, A.E.; Steinkraus, D.C. 2001: Biology, ecology and pest management potential of entomophthorales. In: Butt, T.M.; Jackson, C.; Magan, N. ed. Fungi as biocontrol agents. CAB International, Wallingford, UK. Pp. 71153.

Singh, P. 1983: A general purpose laboratory diet mixture for rearing insects. Insect Science and its Application 4: 253-256.

Yeo, H.; Pell, J.K.; Walter, M.; Boyd-Wilson, K.S.H.; Snelling, C.; Suckling, D.M. 2001: Susceptibility of diamondback moth (Plutella xylostella (L.)) larvae to the entomopathogenic fungus, Zoophthora radicans (Brefeld) Batko. N.Z. Plant Prot. 54: 47-50. 